\title{
A numerical approach for natural overburden stress correction.
}

\author{
Carreira, V. R. ${ }^{1}$; Bijani, S. ${ }^{2}$; Ponte-Neto, C. F. ${ }^{1}$ \\ ${ }^{1}$ Observatório Nacional, ${ }^{2}$ Universidade Federal Fluminense
}

Copyright 2021, SBGf - Sociedade Brasileira de Geofísica.

This paper was prepared for presentation during the 17th International Congress of the Brazilian Geophysical Society held in Rio de Janeiro, Brazil, 16-19 August 2021. Contents of this paper were reviewed by the Technical Committee of the 17th International Congress of the Brazilian Geophysical Society and do not necessarily represent any position of the SBGf its officers or members. Electronic reproduction represtion ritten consent of the Brazilian Geophysical Society is prohibited.

\section{Abstract}

This work aim to define a low cost and low timeconsuming viable alternative for natural overburden correction. Natural overburden is a common displacement observed in sonic and density well logs. Well log data was drilled in Parana Sedimentary Basin. In density channel two tests were performed. First test indicates averages error of $1.24^{-15}$ and second tests performs correction with average error of $1.0071^{-15}$. Difference between original density and calculated density channel was $0.0844 \mathrm{~g} / \mathrm{cm}^{3}$. Tests indicates a feasible methodology for overburden stress correction.

\section{Introduction}

Natural overburden stress is a resulting force, in a stress field acting under a cubic sample of soil or rock, which gradually increases in direction, module and magnitude towards the center of mass of the planet Earth as the exploration depth increases.

Effects of overburden pressure on porosity and permeability lies mainly in clay minerals by reducing primary porosity and generating secondary porosity. The impact on clay minerals is interesting and extremely important due to the existence of chemical and mechanical compaction.

Chemical compaction results in an increase in the kinematic speed of the cation exchange in aqueous solution in the presence of a field of stresses and temperatures greater than 80 and $100^{\circ} \mathrm{C}$ and at depths greater than 2-4 km, in sedimentary basins (Meade, 1966).

Mechanical compaction changes in the eletroacoustic properties of the main clay minerals. Smectite and kaolinite can be peeled as the main clay minerals. Clay minerals such as illite, chlorite and etc., do not have a large cation exchange capacity and small surface area. Smectite is the finest clay mineral found in nature and has a high cation exchange capacity and a large area, while kaolinite has coarser grains and a low cation exchange capacity is a smaller useful area (Mesri and Olson, 1971) and (Rieke and Chilingarian, 1974) apud (Mondol et al., 2007).

Many strategies of natural overburden effect correction has been published over the years as in classical works of Hottmann et al. (1965) and Eaton (1972). Eaton et al.
(1975) defines fours equations that corrects geopressure by calculating directly from resistivity, conductivity and sonic travel-time measurements plots. Jesswein et al. (2018) uses genetic algorithm to correct overburden effect.

We propose a methodology that corrects overburden effect by calculating a $\Phi$ function by predicting the best set of angular and linear coefficients, taking the origin of the Cartesian coordinate system $[x, z]$ or two sets specific ordered pairs called top and bottom $\left(x_{1}, z_{1}\right)$ and $\left(x_{2}, z_{2}\right)$.

\section{Method}

We validate this methodology by applying in real well log data drilled in Parana Sedimentary Basin, south portion of Brazil. Two strategies have been applied. First strategy we apply correction all across the well. In a second strategy we have specify the top and bottom depth that we want to correct predict.

\section{Mathematical considerations}

Let be $\mathbf{d}$ represent $N$ measures of physical properties in a well logging data, where $\mathbf{d}=\left[x_{1}, x_{2}, x_{3}, \ldots, x_{n}\right]$. Depth or vector $\mathbf{p}=\left[z_{1}, z_{2}, z_{3}, \ldots, z_{n}\right]$ is not necessarily physical data, but it does carry information about the geometry.

We assume a model in which the overload adjustment function $\mathrm{S}$ is a linear function of the type $S=a z+b$. The coefficients $(a, b)$ form the parameter model of the problem, $\mathbf{m}=[a b]^{T}$. And according to this model, each property observation must satisfy $S_{i}=a z_{i}+b$. In a sense that,

$$
\begin{gathered}
S_{1}=a z_{1}+b \\
S_{2}=a z_{2}+b \\
S_{3}=a z_{3}+b \\
\vdots \\
S_{n}=a z_{n}+b
\end{gathered}
$$

Equation 1 can be rewritten in the matrix form as $\mathbf{d}=\mathbf{G m}$.

$$
S=\left[\begin{array}{ll}
\frac{\partial S}{\partial a} & \frac{\partial S}{\partial b}
\end{array}\right]\left[\begin{array}{l}
a \\
b
\end{array}\right]
$$

Equation 2 first term, $\mathbf{G}$, is know as matrix kernel matrix, sensitivity matrix or Jacobean matrix. Jacobin matrix is assembled by partially deriving from the geophysical functional parameters. This is a well posed problem in which the rank of the matrix is equal to the number of parameters of the problem. Matrix $\mathbf{G}$ is composed of 
linearly independent vectors. Values of the columns of the sensitivity matrix must be non-zero.

$$
\begin{aligned}
\mathbf{G} & =\left[\begin{array}{ll}
\frac{\partial S}{\partial a} & \frac{\partial S}{\partial b}
\end{array}\right] \\
{\left[\begin{array}{c}
S_{1} \\
S_{2} \\
\vdots \\
S_{N}
\end{array}\right] } & =\left[\begin{array}{cc}
x_{1} & 1 \\
x_{2} & 1 \\
\vdots & 1 \\
x_{N} & 1
\end{array}\right] \quad\left[\begin{array}{l}
a \\
b
\end{array}\right]
\end{aligned}
$$

Least squares solution parameters vector $\boldsymbol{m}$

By taking matrix equation $\mathbf{d}=\mathbf{G m}$, we have

$$
\begin{gathered}
G^{T} d=G^{T} G m \\
m_{e s t}=\left(G^{T} G\right)^{-1} G^{T} d
\end{gathered}
$$

By multiplying $\mathbf{G}$ matrix by its transpose the outcome result is an identity matrix. Matrix division indicated by the negative exponent of the term $\left(G^{T} G\right)^{-1}$. By isolating vector $\mathbf{m}$ we can now calculate $\mathbf{m}_{e s t}$, in equation 3 .

All tests performed on RHOB channel.

\section{Results}

Several authors such as Glover (2003) and Nweke et al. (2016) called into question about how bulk density have it true value changed under overburden pressure influence. Sen et al. (2017) has already noted an inconsistency with Overburden Gradient (OBG) calculated from four differently computed shallow densities.

In an attempt to do RHOB channel correction, we first have applied our methodology in all bulk density data channel. This procedure was chosen because it is one of the most practical and feasible ways to correct channel for all data points. A total of 13431 samples were selected for this first test.

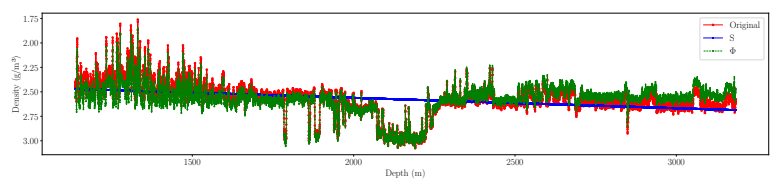

Figure 1: First approach chosen to overburden correction. Green line represent $\phi$ estimated data. Red line presents the original data. Blue line represents the best calculated parameters sets.

Figure 1 indicates that by having applied the numerical overburden stress correction in all data points we have rotated the original data. It can be observed by a displacement of the top and bottom parts of the calculated channel $\phi$ in relation to original channel. Mean error indicates an average of $1.24 \times 10^{-15}$.

For the second test we opted for chose a small sample size. we have created a target limited by a top and a bottom layers. The sample was selected and subdivided in a top layer of depth $1400 \mathrm{~m}$ and bottom layer of depth $1750 \mathrm{~m}$ and a total of 2296 samples.

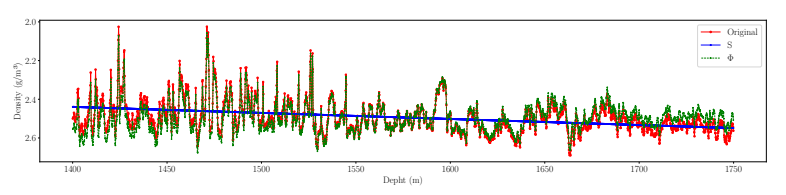

Figure 2: Second approach for overburden correction of a specific target with top depth of $1400 \mathrm{~m}$ and bottom depth of $1750 \mathrm{~m}$. Green line represent $\phi$ estimated data. Red line presents the original data. Blue line represents the best calculated parameters sets

Figure 2 show the windowed data sampled. We observed also a rotation of the fitted curve in relation to the top and the bottom data points. Mean error indicates an average of $1.01 \times 10^{-15}$ for all channel.

For better observe how windowed data fits in relation to complete data set we have analysed all the 13431 samples.

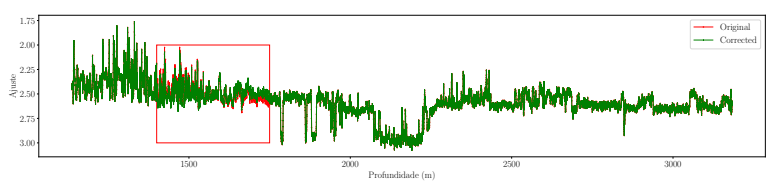

Figure 3: Second approach for overburden correction with all 13431 samples. Red box represents the 2296 data corrected. Red line presents the original data. Green line represents data misfit.

In figure 3 indicates the behavior of corrected data displacement. True densities means indicates averages of $2.57 \mathrm{~g} / \mathrm{cm}^{3}$ and calculated density means indicates average of $2.49 \mathrm{~g} / \mathrm{cm}^{3}$. Average density means present $0.084 \mathrm{~g} / \mathrm{cm}^{3}$ of an average displacement.

\section{Conclusions}

This method represents a low cost and low time-consuming viable alternative for natural overburden correction. However, it indicates a limitation with the linear fit and rotation observed in the corrected data.

First set of analyses investigated behavior of corrected data in relation to the original $\mathrm{RHOB}$ channel. $\mathrm{RHOB}$ calculated channel shows a rotational displacement by calculating data through fitting a straight line. Straight line calculated by inversion of the best set of parameters clearly creates a tendency in calculated data. It is clearly a disadvantage.

In order to minimize the effect of rotational displacement we have conduct a second test. Second test indicates lower values for error comparative to first test. As expected rotational displacement also occurs despite creating a window target for well log data set. Despite rotational displacement occurred by windowing data set sample we could minimize mean error comparatively to the first test. We have concluded that constructed small window samples the error and the rotational displacement is minimized. 


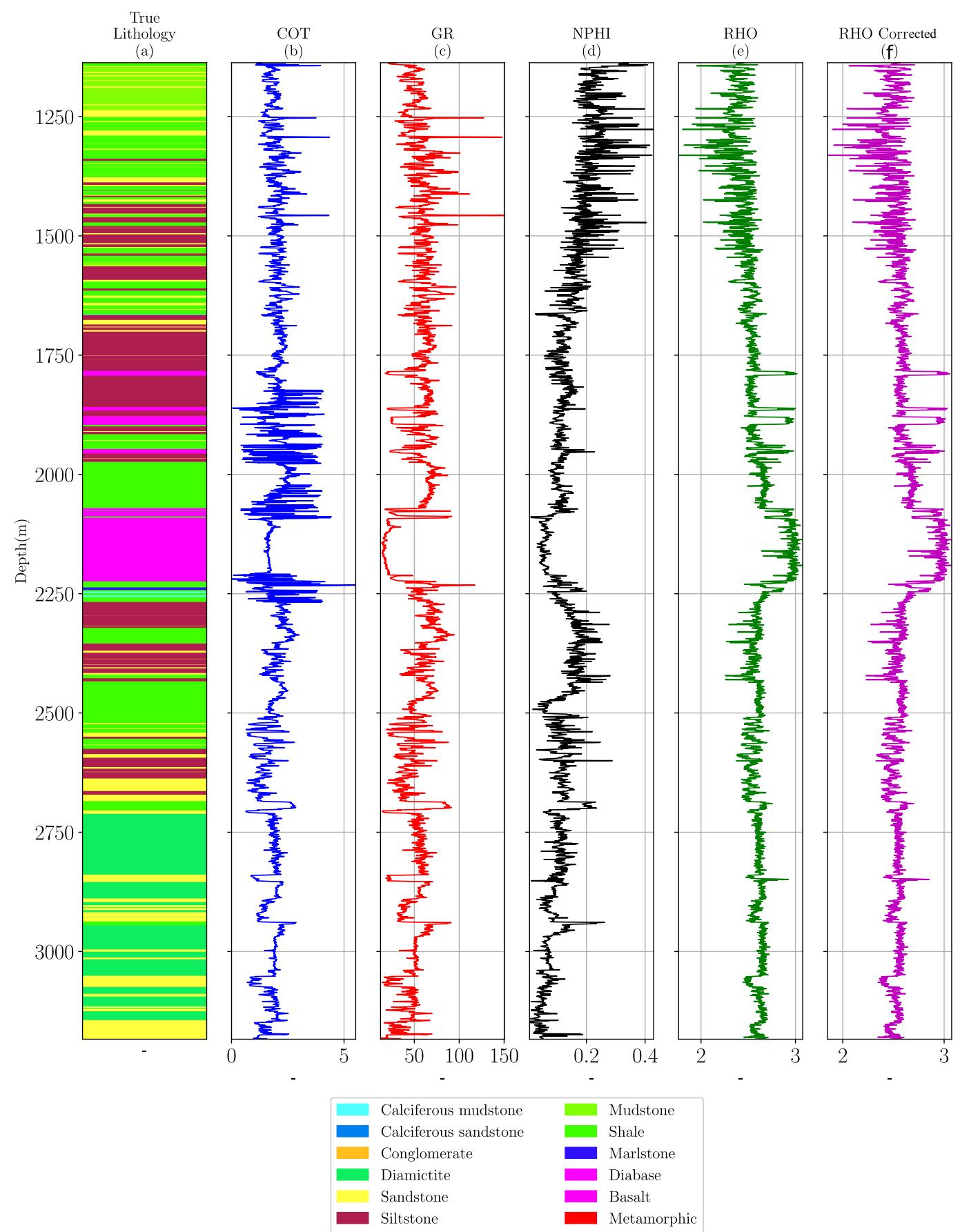

Figure 4: Figure (e) indicates original channel with no correction. Figure (f) indicates corrected RHOB channel. 


\section{Acknowledgments}

Special thanks to Fundação de Amparo à Pesquisa do Estado do Rio de Janeiro (FAPERJ) for financial support.

\section{References}

Eaton, B. A., 1972, The Effect of Overburden Stress on Geopressure Prediction from Well Logs: Journal of Petroleum Technology, 24, 929-934.

Eaton, B. A., et al., 1975, The equation for geopressure prediction from well logs: Presented at the Fall meeting of the Society of Petroleum Engineers of AIME, Society of Petroleum Engineers.

Glover, P. W., 2003, Section 13 - The Formation Density Log: Petrophysics MSc Course Notes, 121-138.

Hottmann, C., R. Johnson, et al., 1965, Estimation of formation pressures from log-derived shale properties: Journal of Petroleum Technology, 17, 717-722.

Jesswein, M., J. Liu, and M. Kwak, 2018, Predicting the side resistance of piles using a genetic algorithm and spt n-values: Presented at the , GeoEdmon - Canada.

Meade, R. H., 1966, Factors influencing the early stages of the compaction of clays and sands; review: Journal of Sedimentary Research, 36, 1085-1101.

Mesri, G., and R. E. Olson, 1971, Mechanisms controlling the permeability of clays: Clays and Clay minerals, $\mathbf{1 9}$, 151-158.

Mondol, N. H., K. Bjørlykke, J. Jahren, and K. Høeg, 2007, Experimental mechanical compaction of clay mineral aggregates-Changes in physical properties of mudstones during burial: Marine and Petroleum Geology, 24, 289-311.

Nweke, I., A. Oriji, and A. Dosunmu, 2016, Development of the Overburden Stress Relationship in Niger Delta Brown Fields: The International Journal Of Engineering And Science, 5, 11-15.

Rieke, H. H., and G. V. Chilingarian, 1974, Compaction of argillaceous sediments: Elsevier.

Sen, S., J. Corless, S. Dasgupta, C. Maxwell, and M. Kumar, 2017, Issues faced while calculating overburden gradient and picking shale zones to predict pore pressure: 1st EAGE Workshop on Pore Pressure Prediction: At the Well Scale from Today to Tomorrow, State of the Art, Recent Progress and Technology Gap. 